\title{
DOENÇA DE MONDOR EM RARA LOCALIZAÇÃO: VEIA EPIGÁSTRICA SUPERFICIAL DIREITA
}

\author{
Leonardo Barros PICCININI ${ }^{*}$, Emanuela de Oliveira GALLO ${ }^{2}$, Antônio Cascelli VAZ FILHO ${ }^{1}$, Daniel \\ Fernandes GUIMARÃES ${ }^{1}$, Ricardo Turra PERRONE ${ }^{1}$, Jaffer Tardim CARDERELLI ${ }^{1}$, Alexandre Funes \\ BASTOS $^{1,2}$ \& Eugênio Carlos de Almeida TINOCO ${ }^{1,2}$
}

1 Hospital São José do Avaí (HSJA), Itaperuna, RJ, Brasil.

2 Universidade Iguaçu, Campus V (UNIG), Itaperuna, RJ, Brasil.

*Autor para correspondência: leopiccinini@ hotmail.com

DOI: http://dx.doi.org/10.18571/acbm.165

\section{RESUMO}

A Doença de Mondor é uma rara tromboflebite de veias do subcutâneo da parede toracoabdominal anterolateral, com maior prevalência no sexo feminino com idade média de 30 a 60 anos, idiopática e autolimitada, podendo estar associada a coagulopatias, traumas e neoplasia de mama. Objetivo: Relatar o caso de um paciente de 38 anos, com Doença de Mondor em veia epigástrica superficial, diagnosticado pelo Serviço de Cirurgia Vascular e Endovascular do Hospital São José do Avaí, Itaperuna, RJ, em março de 2016. Conclusão: Paciente foi tratado conservadoramente e após duas semanas de acompanhamento apresentou recuperação favorável com melhora significativa dos sintomas.

Palavras chave: Doença de Mondor; Tromboflebite Superficial; Veia Epigástrica Superficial.

\begin{abstract}
Mondor's disease is a rare thrombophlebitis of the subcutaneous veins of the anterolateral thoraco-abdominal wall, with a higher prevalence in women with a mean age of 30 to 60 years, idiopathic and self-limited, and may be associated with coagulopathies, traumas and neoplasia of breast. Objective: To report the case of a 38-year-old patient with Mondor's disease in a superficial epigastric vein, diagnosed by the Vascular and Endovascular Surgery Service of the São José do Avaí Hospital, Itaperuna, RJ, in March 2016. Conclusion: Patient was treated conservatively and after two weeks of follow-up presented a favorable recovery with significant improvement of the symptoms.
\end{abstract}

Keywords: Mondor's disease; Superficial Thrombophlebitis; Superficial Epigastric Vein.

\section{Introdução}

Doença de Mondor é descrita como uma rara tromboflebite de veias do subcutâneo da parede toraco-abdominal antero-lateral, com prevalência maior em mulheres, com idade média entre 30-60 anos, na maioria das vezes idiopática, autolimitada e caráter benigno (MAYOR, 2000).

As manifestações clínicas mais comuns são mal-estar, febre, cordão subcutâneo doloroso, sensação de tensão e retração da pele e que desaperecem em média de 2 a 10 semanas após inicio das manifestações (MAYOR, 2000; CATANIA 1992). 


\section{ACTA \\ Biomedica Brasiliensia}

A patogenia da doença ainda é incerta, porém, há relatos na literatura que associam, como possíveis causas, trauma direto, cirurgia, esforço muscular, biópsia, radiação, infecção e câncer de mama (FAUCZ, 2005).

O diagnóstico geralmente é clínico. Mas os achados de imagens têm importante contribuição, pois confirmam que os sintomas correspondem a uma veia superficial trombosada (FAUCZ, 2005).

Descrevemos um caso de doença de Mondor em veia epigástrica superficial direita, relatando a clínica e mostrando os achados de imagem típicos desta enfermidade.

\section{Caso Clínico}

F.B.D.S, 38 anos, sexo masculino, pardo, natural de Itaperuna - RJ, foi atendido em março de 2016 no ambulatório de cirurgia vascular no Hospital São José do Avaí, com queixa de dor abdominal em fossa ilíaca direita e flanco direito até nível de arcos costais, iniciada há 2 meses. O mesmo referia piora dos sintomas após esforço físico vigoroso. Relatou que desde a infância apresentava episódios com quadro clínico semelhante, em paroxismos, de mesma topografia. Procurou atendimento no HSJA devido à hipótese diagnóstica de hérnia inguinal. Ao exame físico observou-se massa em região inguinal direita e cordão endurado com trajeto ascendente, em subcutâneo, associado a sinais flogísticos. Através do exame de angiotomografia computadorizada foi evidenciada veia epigástrica superficial tortuosa (figuras 1A e 1B) e de calibre aumentado (figura 2). Para melhor avaliação e medida do diâmetro da lesão foi realizada reconstrução 3D (figura 3). A partir do laudo da angiotomografia, o paciente foi orientado ao uso de medicação sintomática, com compressas quentes, analgésico e anticoagulação com rivaroxabana. Foi solicitado exames para pesquisa de trombofilias. Após duas semanas de tratamento paciente retornou ao serviço apresentando ótima evolução do quadro e regressão dos sinais flogísticos. Resultado para trombolifias apresentou-se negativo.

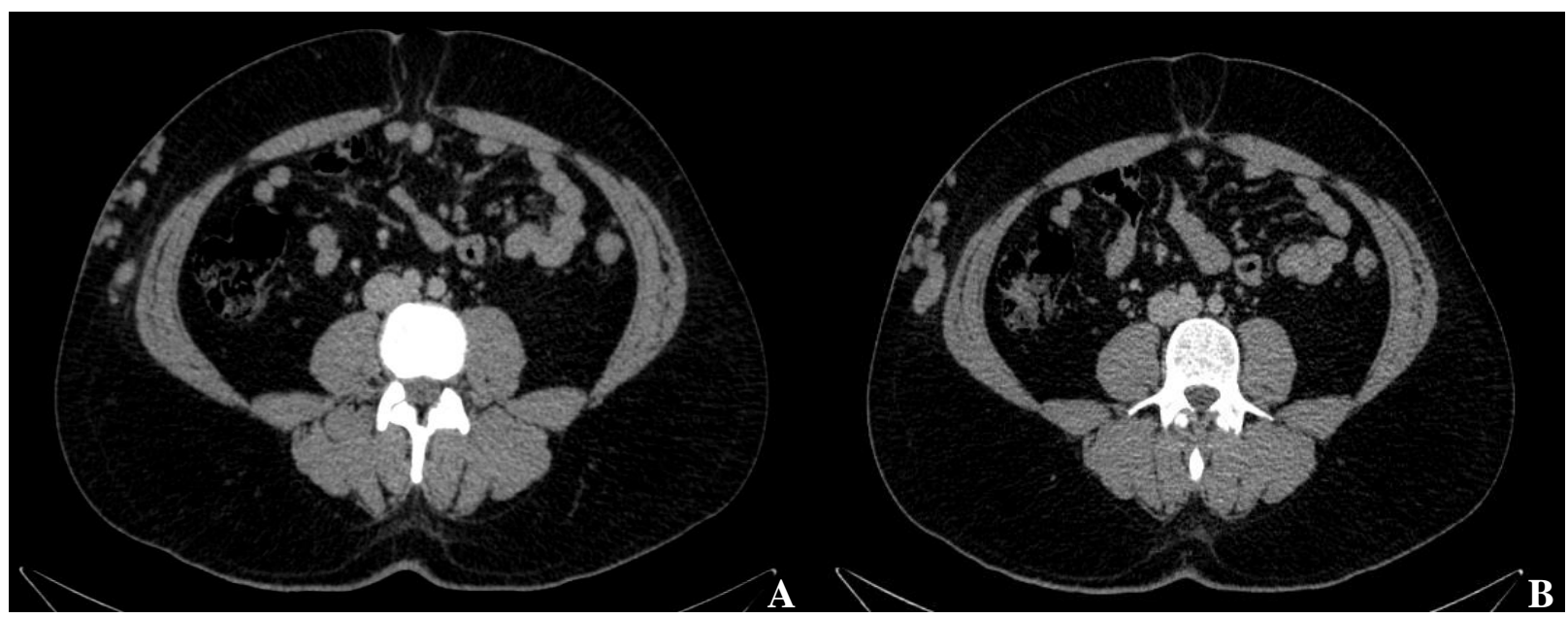

Figuras 1 (A e B): Angiotomografia computadorizada evidenciando tromboflebite de veia epigástrica superficial. 


\section{ACTA}

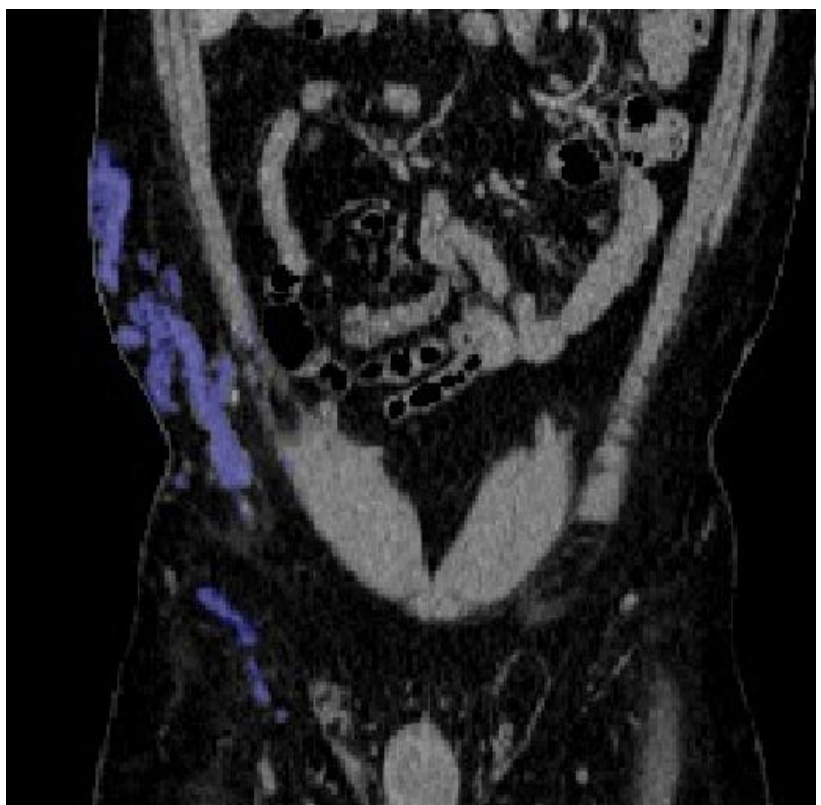

Figura 2: Área em azul, evidenciando lesão.

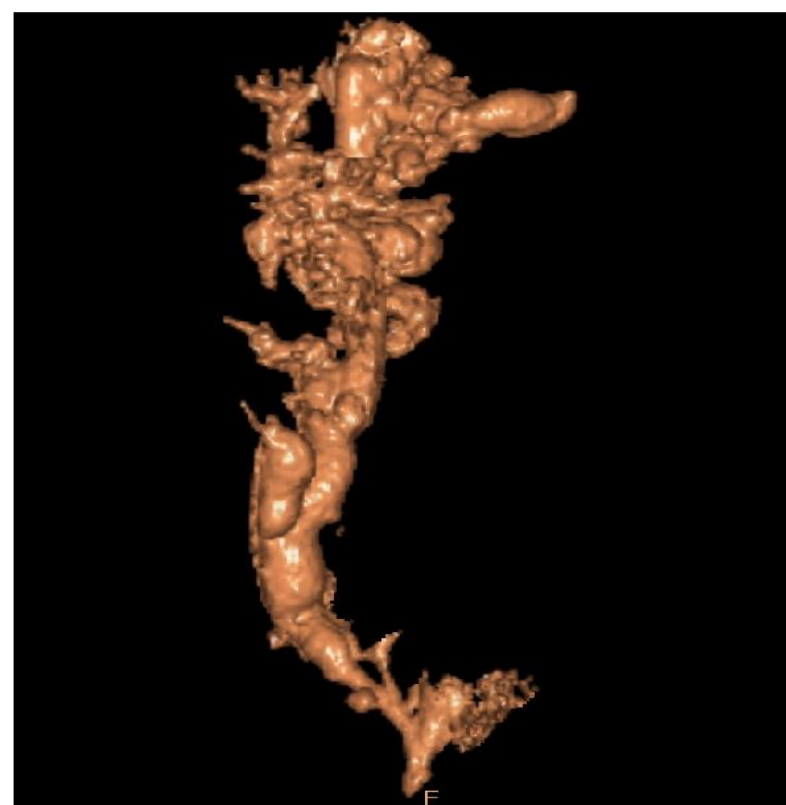

Figura 3: Reconstrução 3D do segmento acometido.

\section{Discussão}

A doença de Mondor é uma patologia rara, descrita pela primeira vez por Henri Mondor em 1939, caracterizada por tromboflebite dos vasos superficiais da região toracoabdominal. (COPPETTI, 2012).

Sua etiologia ainda não está bem definida, porém há relatos na literatura que associam, como possíveis causas, traumas diretos, cirurgias, biopsia, esforço muscular, radiação, infecção e câncer de mama (FAUCZ, 2005).

Frequentemente a prevalência é maior em mulheres de meia idade e normalmente os vasos mais acometidos são segmentos da veia tóraco-epigástrica, veia torácica lateral e veia epigástrica superior (SHETTY, 2001 FIETTA 2002).

A Doença de Mondor, que embora rara, possui caráter benigno e autolimitado (MAYOR, 2000).

Embora o diagnóstico habitualmente seja clinico pela presença de um cordão superficial na região torácica, ele pode ser confirmado através de exames de imagens. (PINTO, 2014).

O tratamento é conservador e sintomático com medidas que visem a redução da dor como repouso, calor local, analgésicos. Anti-inflamatórios não asteroidais e anticoagulantes podem ser prescritos em situações mais complexas (COPPETTI, 2012).

A importância do relato deste caso é demonstrar uma forma rara de acometimento da doença de Mondor, em relação à topografia da lesão (veia epigástrica superficial) e ao sexo (masculino) e reforçar que mesmo em localização rara, o tratamento é sintomático e a doença possui curso benigno.

\section{Conclusão}

A doença de Mondor com acometimento de topografia não usual obteve sucesso com o tratamento preconizado na literatura. Até o momento o paciente encontra-se em remissão dos sintomas, não apresentando comprometimento na qualidade de vida. Deve-se realizar uma 
investigação complementar minuciosa nos casos onde se suspeite de malignidade ou doença sistêmica associada.

Mais estudos são necessários para avaliar possíveis complicações e malignização no seguimento de pacientes com Doença de Mondor em topografia não usual.

\section{Referências}

CATANIA S, ZURRIDA S VERONESI P, GALIMBERT V, BONO A, PLUCHINOTTA A. Mondor's disease and breast cancer. Cancer 1992;69:2267-70.

COPETTI JM, COPETTI FV, NUNES AG. Doença de Mondor associada à cirurgia de ginecomastia e do mamilo. Resvita Brasileira de Cirurgia Plástica. Vol. 27 nº 4 - Out/Nov/Dez de 2012

FAUCZ RA, HIDALGO RT, FAUCZ RS. Doença de Mondor: achados mamográficos e ultrasonográficos. Radiol Bras. 2005; 38(2):153-5.

FIETTA, P, MANGANELLI P. Mondor's diseases. Spectrum of the clinical and pathological features. Minerva Med ,2002; 93:453-6.

MAYOR, M.; BURÓN I.; MORA JC.; LÁZARO TE.; HERNÁNDEZ-CANO N.; RUBIO FA.; et al. Mondor's disease. Int J Dermatol 2000; 39:922-5.

PINTO, Rita Mesquita et al. Mondor's disease in puerperium: case report. Rev. Bras. Ginecol. Obstet. Rio de Janeiro, v. 36, n. 3, p. 139-141, Mar. 2014.

SHETTY MK, WATSON AB. Mondor's disease of the breast: sonographic and mammographic findings. American Journal of Roentgenology. 2001; 177:893-6 